\title{
Osteonecrosis de los maxilares asociada a bifosfonatos: revisión sistemática
}

\section{Escobar López EA*, López López J**, Marques Soares MS***, Chimenos Küstner E**}

\section{RESUMEN}

En los últimos años se ha descrito una nueva complicación en la terapia con bifosfonatos: la osteonecrosis de los maxilares. Esta complicación no había sido detectada en los ensayos clínicos previos. En esta revisión sistemática de 340 casos, los pacientes afectados presentan como diagnóstico primario principalmente: mieloma múltiple $(51,2 \%)$, cáncer de mama $(31,4 \%)$, cáncer de próstata $(7,1 \%)$ y osteoporosis $(4,1 \%)$. La mandíbula resulta más afectada que el maxilar, con el 59,1\%. La relación entre género femenino y masculino es de 2:2,6. El pamidronato es el bifosfonato más asociado a los casos de osteonecrosis (35,3\%). Los factores de riesgos desencadenantes incluyen: exposición a bifosfonatos, infecciones orales, traumas, procedimientos quirúrgicos orales previos y terapias contra el cáncer (quimioterapia, corticoides y otros agentes citostáticos). La alteración en el equilibrio óseo, factores antiangiogénicos, inhibición en el ciclo celular de los queratinocitos y mecanismos osteolíticos generados en la infección podrían explicar la etiopatogenia en el desarrollo de esta complicación.

Palabras clave: Osteonecrosis, bifosfonatos, maxilares, pamidronato, ácido zoledrónico.

\section{SUMMARY}

In the last years a new complication has been described in the therapy with biphosphonates: biphosphonateassociated osteonecrosis of the jaws. This complication had not been detected in previous clinical tests. In this systematic review of 340 cases, the patients had as primary diagnosis principally: multiple myeloma (51.2\%), breast cancer $(31.4 \%)$, prostate cancer $(7.1 \%)$ and osteoporosis $(4.1 \%)$. The mandible is more affected than the maxilla, with $59.1 \%$. The relationship between femenine and masculine gender was 2:2.6. The pamidronate was the biphosphonate more associated to the cases of osteonecrosis (35.3\%). The risk factors associated include: use of biphosphonates, presence of oral infections, trauma, oral surgical procedures and therapies against cancer (chemoterapy, corticosteroids and other cytostatics agents). Effects on bone turnover, antiangiogenic property of biphosphonates, inhibitory effect of biphosphonates on the keratinocyte cell cycle and osteolysis mechanisms induced in the bacterial infection could explain the etiological mechanisms in the development of this complication.

Key words: Osteonecrosis, bisphosphonates, jaws, pamidronate, zoledronate.

Fecha de recepción: Septiembre 2006.

Aceptado para publicación: Diciembre 2006.

* Diploma de Postgrado de Medicina Bucal, Facultad de Odontología, Universidad de Barcelona.

** Profesor Titular de Medicina Bucal, Facultad de Odontología, Universidad de Barcelona.

*** Profesora Titular de Medicina Bucal, Universidad Federal de Paraíba (Brasil).

Escobar López EA, López López J, Marques Soares MS, Chimenos Küstner E. Osteonecrosis de los maxilares asociada a bifosfonatos: revisión sistemática. Av. Odontoestomatol 2007; 23 (2): 91-101. 


\section{INTRODUCCIÓN}

Anteriormente al año 2003 se habían descrito en la literatura osteonecrosis de los maxilares como complicación terapéutica en pacientes en tratamiento por neoplasias y tumores malignos asociadas a la quimioterapia $^{1,2}$ y radioterapia ${ }^{3,4}$. A partir del año 2003 se comenzaron a publicar en la literatura casos y series de pacientes afectados principalmente con mieloma múltiple y cáncer de mama, que presentaban osteonecrosis de los maxilares y que tenían como patrón común en la terapia farmacológica el uso de bifosfonatos intravenosos: pamidronato $90 \mathrm{mg}$ (Aredia ${ }^{\circledR}$ ) y ácido zoledrónico 4 mg (Zometa ${ }^{\circledR}$ ).

Las primeras series de esta complicación fueron publicadas ese mismo año: Marx ${ }^{5}$ (36 casos), Wang et al. ${ }^{6}$ (3 casos) y Migliorati ${ }^{7}$ (5 casos). A pesar de que ya se conocían reacciones adversas al uso de bifosfonatos $^{8}$, la osteonecrosis de los maxilares no había sido detectada en los ensayos clínicos previos a la comercialización de estos fármacos.

Los bifosfonatos son análogos sintéticos de los pirofosfatos inorgánicos y tienen una alta afinidad por el calcio. Son potentes inhibidores de la reabsorción osteoclástica. Difieren de los pirofosfatos endógenos por presentar una cadena central P-O-P, resultante de la sustitución de un átomo de carbono (P$\mathrm{C}-\mathrm{P}$ ) por oxígeno y variando las cadenas laterales $\mathrm{R} 1$ y R2. La unión a los cristales de hidroxiapatita es realizada en R1. La cadena R2 y su estructura tridimensional determinan los efectos a nivel celular de los bifosfonatos y su eficacia relativa como inhibidores de la reabsorción ósea, otorgándole un perfil de actividad característico para cada bifosfonato ${ }^{9}$. Entre las propiedades biológicas y los mecanismos de acción de los bifosfonatos se encuentran los siguientes: 1) inhibición de la reabsorción ósea osteoclásti$\mathrm{ca}^{10}$; 2) inducción de los osteoblastos a secretar un inhibidor de la secreción ósea mediada por osteoclastos $^{11}$; 3) inducción de apoptosis en los osteoclastos $^{12}$; 4) inhibición de la apoptosis de osteocitos y células de la línea osteoblástica ${ }^{13}$, y 5) efecto antiangiogénico que reduce el EGF (factor de crecimiento endotelial) $)^{14}$. Además, los bifosfonatos presentan propiedades antitumorales: inhibición de la proliferación e invasión de las células tumorales ${ }^{15}$; inhibición de la adhesión de las células tumorales al tejido óseo ${ }^{16}$; inducción de apoptosis en varias líneas celulares malignas ${ }^{17}$ e inhibición de la actividad proteolítica de las metaloproteinasas de la matriz ${ }^{18}$.

Se emplean en el tratamiento de lesiones osteolíticas en el mieloma múltiple, en metástasis óseas predominantemente líticas (especialmente en el cáncer de mama y de próstata) y en la hipercalcemia maligna inducida por tumores. Se utilizan también en el tratamiento de osteoporosis: en post menopausia, en hombres y en las inducidas por glucocorticoides. Tienen asimismo indicación terapéutica en la enfermedad de Paget, osteogénesis imperfecta y otras enfermedades metabólicas óseas ${ }^{9}$.

Clínicamente ${ }^{19,20}$ las lesiones a nivel intraoral se presentan como áreas únicas o múltiples de exposición de hueso necrótico al medio bucal. Otros signos y síntomas incluyen: dolor, movilidad dentaria, tumefacción, supuración purulenta, parestesia en el territorio del nervio dentario inferior. A nivel extraoral se puede observar la presencia de fístulas y aumento de volumen de los tejidos blandos de la región cervicofacial.

En este artículo se presentan los resultados de una revisión sistematizada sobre osteonecrosis de los maxilares asociada a bifosfonatos, considerándose edad, género, diagnóstico primario, tipo de bifosfonato, localización anatómica y procedimientos odontológicos previos.

\section{MATERIAL Y MÉTODO}

Se realizó una revisión bibliográfica en MEDLINE y PubMed, de septiembre 2003 a mayo 2006, que incluyeran los siguientes términos en lengua inglesa: osteonecrosis, avascular necrosis, bis o biphosphonates, y se cruzaron estos términos con los siguientes: jaw, cancer, myeloma. Se incluyeron 62 publicaciones en idioma inglés y una en idioma alemán, totalizando 63 publicaciones que documentaban información de casos o series de casos relacionadas con osteonecrosis o necrosis avascular de los maxilares asociada a bifosfonatos o relacionadas a estos. La información se consignó según edad, género, diagnóstico primario, tipo de bifosfonato, localización anatómica y procedimientos odontológicos previos. 


\section{RESULTADOS}

Se recogen 340 casos de osteonecrosis de los maxilares asociada a bifosfonatos (ONB). Se contabilizaron 63 publicaciones (Tabla 1) desde septiembre 2003 hasta mayo 2006. El rango de edad fue de 36 a 92 años y el promedio de edad de 65,5 años. El pico de edad se localizó entre la séptima y la octava década (Tabla 2). El género más afectado fue el femenino $(47,9 \%)$ (Tabla 3), en una relación de 2:2,6 con respecto al masculino.

\begin{tabular}{|lrr|llr|}
\hline \multicolumn{5}{|c|}{ TABLA 1.- } & SERIES DE CASOS CON \\
OSTEONECROSIS DE LOS MAXILARES, \\
ASOCIADOS
\end{tabular}

\begin{tabular}{|lrr|}
\hline \multicolumn{3}{|c|}{$\begin{array}{c}\text { TABLA 2.- DISTRIBUCIÓN POR } \\
\text { DÉCADAS DE LA ONB }\end{array}$} \\
\hline & $\mathbf{n}$ & \multicolumn{1}{c|}{$(\%)$} \\
\hline $31-40$ & 3 & $(0,9)$ \\
$41-50$ & 23 & $(6,8)$ \\
$51-60$ & 51 & $(15,0)$ \\
$61-70$ & 70 & $(20,6)$ \\
$71-80$ & 64 & $(18,8)$ \\
$81-90$ & 16 & $(4,7)$ \\
$91-100$ & 1 & $(0,3)$ \\
Sin información & 112 & $(32,9)$ \\
\hline Total & 340 & $(100,0)$ \\
\hline
\end{tabular}

El mieloma múltiple fue el diagnóstico primario más frecuente con el $51,2 \%$ (Tabla 4). El pamidronato fue el bifosfonato más utilizado (35,3\%) (Tabla 5). La mandíbula resultó el hueso más comprometido (Tabla 6). El

\section{TABLA 3.- DISTRIBUCIÓN POR GÉNERO DE LA ONB}

\begin{tabular}{|lrl|}
\hline & $\mathbf{n}$ & $\mathbf{( \% )}$ \\
\hline Femenino & 163 & $(47,9)$ \\
Masculino & 127 & $(37,4)$ \\
Sin información & 50 & $(14,7)$ \\
\hline Total & 340 & $(100,0)$ \\
\hline
\end{tabular}

\begin{tabular}{|c|c|c|}
\hline \multicolumn{3}{|c|}{$\begin{array}{c}\text { TABLA 4.- DIAGNÓSTICO PRIMARIO } \\
\text { ASOCIADO A ONB }\end{array}$} \\
\hline & $\mathrm{n}$ & (\%) \\
\hline Mieloma múltiple & 174 & $(51,2)$ \\
\hline Carcinoma mama & 107 & $(31,4)$ \\
\hline Carcinoma próstata & 24 & $(7,1)$ \\
\hline Osteoporosis & 14 & $(4,1)$ \\
\hline Otros & 8 & $(2,3)$ \\
\hline Carcinoma pulmón & 5 & $(1,5)$ \\
\hline Carcinoma renal & 4 & $(1,2)$ \\
\hline Enfermedad de Paget & 4 & $(1,2)$ \\
\hline Total & \multicolumn{2}{|c|}{$340(100,0)$} \\
\hline
\end{tabular}

\section{TABLA 5.- TIPO DE BIFOSFONATOS ASOCIADO A ONB}

\begin{tabular}{|lrr|}
\hline & $\mathbf{n}$ & $\mathbf{( \% )}$ \\
\hline Pamidronato & 120 & $(35,3)$ \\
Ácido zoledrónico & 109 & $(32,0)$ \\
Pamidronato y ácido zoledrónico & 90 & $(26,5)$ \\
Alendronato & 13 & $(3,8)$ \\
Alendronato y ácido zoledrónico & 2 & $(0,6)$ \\
Ibandronato y ácido zoledrónico & 2 & $(0,6)$ \\
Clodronato & 1 & $(0,3)$ \\
Ibandronato & 1 & $(0,3)$ \\
Pamidronato, ibandronato & 1 & $(0,3)$ \\
y ácido zoledrónico & 1 & $(0,3)$ \\
Risendronato & 340 & $(100,0)$ \\
\hline Total & \multicolumn{3}{c}{} \\
\hline
\end{tabular}


AVANCES EN ODONTOESTOMATOLOGÍA

Vol. 23 - Núm. 2 - 2007

TABLA 6.- LOCALIZACIÓN ASOCIADA A ONB

\begin{tabular}{|lrr|}
\hline & $\mathbf{n}$ & (\%) \\
\hline Mandíbula & 200 & $(59,1)$ \\
Maxilar & 71 & $(20,6)$ \\
Ambos & 28 & $(8,2)$ \\
Sin información & 41 & $(12,1)$ \\
\hline Total & 340 & $(100,0)$ \\
\hline
\end{tabular}

ácido zoledrónico se utilizó principalmente en la terapia del cáncer de mama, en tanto que el pamidronato lo fue en el mieloma múltiple (Tabla 7). Se presentaron un $62,9 \%$ de exodoncias previas a la osteonecrosis (Tabla 8) y de éstas el $63,6 \%$ se localizaron en la mandíbula.

\section{DISCUSIÓN}

\section{Prevalencia de la osteonecrosis de los maxilares asociada a bifosfonatos}

Se han publicado numerosos casos y series al respecto, desde el año 2003 hasta la fecha; sin embar-

\begin{tabular}{|lrr|}
\hline \multicolumn{3}{|c|}{ TABLA 8.- PORCENTAJE DE } \\
PROCEDIMIENTOS DENTALES PREVIOS A ONB \\
\hline & $\mathbf{n}$ & $\mathbf{( \% )}$ \\
\hline Exodoncia & 214 & $(62,9)$ \\
Prótesis desajustada & 3 & $(0,9)$ \\
Espontánea & 123 & $(36,2)$ \\
\hline Total & 340 & $(100,0)$ \\
\hline
\end{tabular}

go, la incidencia de esta complicación parece ser baja. En el sur de Australia ${ }^{29}$, en el año 2003, aproximadamente 14.000 pacientes recibían prescripciones médicas para bifosfonatos de segunda y tercera generación que contenían nitrógeno; un 10\% de estos en administración intravenosa, con tan solo 5 casos de osteonecrosis hasta el momento. En el estudio de Durie et al. ${ }^{55}$, en el 2005, se evaluó la incidencia de osteonecrosis asociada a los bifosfonatos en 1.203 pacientes, quienes recibían terapia para mieloma múltiple (904) y cáncer de mama (299). De los 904 pacientes con mieloma múltiple, 62 tuvieron el diagnóstico de osteonecrosis y 54 sospecha de osteonecrosis, resultando un total de 116 pacientes

\section{TABLA 7.- DISTRIBUCIÓN POR TIPO DE BIFOSFONATOS Y DIAGNÓSTICO PRIMARIO EN ONB}

\begin{tabular}{|c|c|c|c|c|c|c|c|c|}
\hline & \multicolumn{2}{|c|}{ Pamidronato } & \multicolumn{2}{|c|}{ Ác. zoledrónico } & \multicolumn{2}{|c|}{$\begin{array}{l}\text { Pamidronato y } \\
\text { ác. zoledrónico }\end{array}$} & \multicolumn{2}{|c|}{ Alendronato } \\
\hline & n & (\%) & $\mathbf{n}$ & (\%) & $\mathbf{n}$ & (\%) & $\mathrm{n}$ & (\%) \\
\hline Mieloma múltiple & 76 & $(63,4)$ & 39 & $(35,8)$ & 57 & $(63,4)$ & & \\
\hline Cáncer de mama & 31 & $(25,9)$ & 47 & $(43,1)$ & 26 & $(28,9)$ & & \\
\hline Cáncer de próstata & 4 & $(3,3)$ & 16 & $(14,7)$ & 3 & $(3,3)$ & & \\
\hline Cáncer de riñón & 2 & $(1,7)$ & 1 & $(0,9)$ & 1 & $(1,1)$ & & \\
\hline Enfermedad de Paget & 2 & $(1,7)$ & & & & & 2 & $(15,4)$ \\
\hline Cáncer de pulmón & 1 & $(0,8)$ & 4 & $(3,7)$ & & & & \\
\hline Leucemia mieloide crónica & 1 & $(0,8)$ & & & & & & \\
\hline Plasmacitoma & 1 & $(0,8)$ & & & & & & \\
\hline Sarcoma uterino & 1 & $(0,8)$ & & & & & & \\
\hline Mesotelioma pleural & 1 & $(0,8)$ & & & & & & \\
\hline Linfoma no Hodgkin & & & & & 1 & $(1,1)$ & & \\
\hline Cáncer ovárico & & & & & 1 & $(1,1)$ & & \\
\hline Osteoporosis & & & & & 1 & $(1,1)$ & 11 & $(84,6)$ \\
\hline Cáncer neuroendocrino & 1 & $(0,9)$ & & & & & & \\
\hline Displasia fibrosa & 1 & $(0,9)$ & & & & & & \\
\hline Total & 120 & $(100,0)$ & 109 & $(100,0)$ & 90 & $(100,0)$ & 13 & $(100,0)$ \\
\hline
\end{tabular}


Escobar López EA, López López J, Marques Soares MS, Chimenos Küstner E.

(12,8\%). De los 299 pacientes con cáncer de mama, 13 pacientes desarrollaron osteonecrosis y 23 sospecha de osteonecrosis, resultando un total de 36 pacientes (12\%). Bamias et al. ${ }^{42}$, en 252 pacientes tratados con bifosfonatos intravenosos, observaron que el 10\% de 111 pacientes con mieloma múltiple y el 3\% de 46 pacientes con cáncer de mama desarrollaron osteonecrosis. En otro estudio, Estilo et al. ${ }^{56}$, en 124 pacientes tratados con bifosfonatos intravenosos con mieloma múltiple y cáncer de mama, 4 y 9 pacientes respectivamente desarrollaron osteonecrosis. Tarassoff et al. ${ }^{57}$, por otro lado, señalan que desde la introducción al mercado de pamidronato y ácido zoledrónico, 2,5 millones de pacientes han recibido terapia con éstos.

\section{Revisión de los casos}

Anteriormente a la aparición de la osteonecrosis de los maxilares como complicación a la terapia con bifosfonatos, se había informado en la literatura que los bifosfonatos orales (alendronato) podrían causar úlceras en la mucosa oral ${ }^{52,53,54}$. Los primeros artículos publicados en la literatura internacional respecto a esta nueva reacción adversa son del año 2003 y corresponden a Marx ${ }^{5}$, Wang ${ }^{6}$ y Migliorati ${ }^{7}$. Marx ${ }^{5}$ informó sobre 36 casos de pacientes que tenían en común haber sido tratados con pamidronato o ácido zoledrónico, 18 de los cuales recibieron estos fármacos por indicación de hipercalcemia relacionada con mieloma múltiple, 17 por hipercalcemia relacionada por metástasis de carcinoma mamario y un paciente por osteoporosis. 22 pacientes (61\%) recibieron además dexametasona, 24 pacientes (67\%) recibieron quimioterapia y 4 pacientes (11\%) habían recibido radioterapia. La mayoría de los pacientes presentaron exposición ósea con dolor, 29 casos $(80,5 \%)$ en la mandíbula, 5 casos en el maxilar (14\%) y 2 casos en ambos huesos (5,5\%). En 28 casos la exodoncia previa fue el desencadenante más frecuente $(77,7 \%)$; los restantes 8 casos $(22,3 \%)$ desarrollaron exposición ósea de manera espontánea. Wang et al. ${ }^{6}$, en 2003, describieron 3 pacientes con cáncer de mama en tratamiento con bifosfonatos (pamidronato) y quimioterapia, con 2 casos en el maxilar y uno en mandíbula; sin embargo, no relacionaron el uso de los bifosfonatos con la osteonecrosis, ya que no existían estudios publicados previamente que describieran esta asociación. Migliorati ${ }^{7}$ informó de 5 pacientes: 3 desarrollaron osteonecrosis de manera espontánea en relación a la línea milohioidea interna y 2 en la región molar mandibular después de exodoncias.

En esta revisión de casos de la literatura, la mandíbula fue la más afectada $(59,1 \%)$. En las series que comprenden 10 o más casos, todos los autores coinciden en que la mandíbula es el hueso más afectado, con osteonecrosis en porcentajes que varían entre el $30,8 \%$ y el $83,3 \% 5,21,25,26,32,33,35,42,43,45,46,49$.

El mieloma múltiple fue el diagnóstico primario más frecuente, con el $51,2 \%$, al igual que en las series de Ruggiero et al. ${ }^{21}$ (46,0\%), Bamias et al. ${ }^{42}$ (64,7\%), Zarychanski et al. ${ }^{49}$ (83,3\%) y Badros et al. ${ }^{45}$ (100\%), entre otros.

Si bien el cáncer de mama $(31,4 \%)$ ocupó el segundo lugar en frecuencia entre los diagnósticos primarios, es el primero en frecuencia entre las series de Migliorati et al. ${ }^{33}$ (55,6\%), Bagán et al. ${ }^{26}(50 \%)$ y Pires et al. ${ }^{25}(50 \%)$.

El género femenino fue el más afectado según esta revisión (47,9\%); sin embargo, en algunas series de 10 o más casos el más afectado fue el masculino: Pires ${ }^{25}$ et al. (75\%), Bamias et al..$^{42}$ (58,8\%), Melo et al. ${ }^{32}(63,6 \%)$, Zarychanski et al. ${ }^{49}(58,3 \%)$, Purcell et al. ${ }^{35}(53,8 \%)$ y Badros et al..$^{45}(77,3 \%)$.

Si bien la mayoría de los casos están asociados a bifosfonatos intravenosos, como pamidronato y ácido zoledrónico o la combinación de ambos, con un $93,8 \%$, lo que significa un alto porcentaje de riesgo en desarrollar osteonecrosis en asociación con estos fármacos, ya se ha descrito en la literatura la aparición de osteonecrosis asociada a bifosfonatos orales, como el alendronato $(3,8 \%)^{21,29,33,35,46}$, especialmente en el tratamiento de la osteoporosis, el risendronato ${ }^{21}$ y el ibandronato ${ }^{30}$. También se ha descrito osteonecrosis asociada al clodronato ${ }^{39}$.

Ruggiero et al. ${ }^{21}$ señalan que en 7 casos de osteonecrosis relacionada con osteoporosis, de un total de 63 pacientes, no existían antecedentes de lesiones malignas ni quimioterapia. Farrugia et al. ${ }^{46}$ en su serie (5 pacientes de 23 en total), ninguno presentó co- 
morbilidad con otros fármacos. En cuanto a la dosis, las indicadas en oncología son frecuentemente 12 veces mayores a las usadas en el tratamiento de la osteoporosis ${ }^{58}$.

En cuanto al tiempo de exposición a los bifosfonatos, Bamias et al. ${ }^{42}$ presentan un rango de 4 a 86 meses, con un promedio de 20 meses, siendo el mieloma múltiple la patología más expuesta, con un rango de 4,5 a 86 meses y un promedio de 28,4 meses, y la terapia pamidronato y ácido zoledrónico la de más larga duración, con un rango de 9,4-86 meses y un promedio de 40,2 meses.

Gibbs et al. ${ }^{27}$ publican un rango de 6 a 66 meses, con un promedio de 22 meses. Bagán et al. ${ }^{26}$ presentan un rango de 10 a 59 meses, con un promedio de 27,3 meses. Carter et al. ${ }^{29}$, en 2005, presentan un rango de 6 a 60 meses, con un promedio de 22,8 meses. En la serie de Melo et al. ${ }^{32}$, la duración de la terapia promedio de bifosfonatos fue de 34 meses. Así pues podemos decir que pacientes que han estado en terapia con bifosfonatos durante más de 6 meses presentan un alto riesgo asociado de osteonecrosis ${ }^{21}$. El uso prolongado en la terapia con bifosfonatos puede provocar microlesiones acumulativas en la estructura ósea mineralizada ${ }^{59}$.

Los pacientes afectados de osteonecrosis de los maxilares asociada a bifosfonatos presentan ciertas similitudes clínicas e histológicas con las asociadas a quimioterapia y radioterapia. La osteonecrosis inducida por quimioterapia es relativamente poco frecuente y se asocia principalmente a la terapia de neoplasias hematológicas malignas, especialmente linfomas ${ }^{1,2}$. Entre los agentes quimioterapéuticos presentes en asociación a la terapia de bifosfonatos se hallan: metotrexato, ciclofosfamida, 5-fluoruracilo, melfalan, vincristina, espirubicina, vinorelbina, paclitaxel, tamoxifeno y doxetacel, entre otros $25,29,30,31,33$.

La osteorradionecrosis, a diferencia de la osteonecrosis asociada a bifosfonatos, raramente compromete los maxilares (menos del $5 \%$ de los casos) y es más común en hombres que en mujeres ${ }^{3,4}$. A diferencia de la osteonecrosis asociada a bifosfonatos, todos los casos contabilizados en esta revisión presentaban una predilección por los maxilares, con excepción de un caso localizado en el conducto auditivo izquierdo ${ }^{60}$.

Una explicación de la mayor propensión de los maxilares que la de otros huesos puede obedecer a que aquéllos se encuentran relacionados a través del ligamento periodontal con los dientes; esta suerte de comunicación, de la cual están separados por no más de 0,5 mm de tejido conectivo denso, hace posible que los mecanismos y vías de propagación de las infecciones de origen odontógeno y periodontal marginal sigan en muchos casos la vía ósea, de tal manera que los huesos maxilares están directamente expuestos a procesos infecciosos relacionados a los dientes (necrosis pulpar séptica y procesos periapicales, agudos o crónicos) y de sus tejidos blandos de sostén (enfermedad periodontal), siendo esta condición de "exclusividad" en relación a los otros huesos, que obviamente no poseen dientes. También factores como la delgadez de la mucosa de revestimiento de los maxilares la hace susceptible a distintos factores traumáticos (por ejemplo: prótesis desajustadas), provocando lesiones ulcerativas y estableciendo una vía de comunicación entre el medio bucal y el hueso, incrementando la posibilidad de osteonecrosis en esa zona. Otro factor anatómico que podría explicar la especial predilección por la mandíbula, sería la irrigación vascular, de tipo terminal.

Todos estos factores vienen a determinar una estructura ósea hipodinámica y biomecánicamente disminuida, en la cual la inhibición de la reabsorción y la alteración funcional en la actividad osteoblástica en la formación de tejido óseo nuevo altera el equilibrio en la dinámica aposición-reabsorción, afectando el metabolismo óseo de manera directa o indirecta. La función osteoclástica es parte fundamental de este ciclo, ya que al reabsorberse matriz ósea mineralizada se liberan citoquinas tales como proteínas morfogenéticas y factores de crecimiento similares a insulina 1 y 2 (ILG1 y ILG2) ${ }^{5}$, los cuales inducen a células totipotenciales mesenquimáticas y osteoblastos premitóticos a diferenciarse en osteoblastos y formar tejido óseo nuevo. La interrupción de este ciclo homeostático por la interrupción de la reabsorción ósea resulta en la acumulación de osteocitos no vitales y en microfracturas de la matriz ósea mineralizada ${ }^{61}$. 
Escobar López EA, López López J, Marques Soares MS, Chimenos Küstner E.

También hay evidencia del efecto inhibitorio de los bifosfonatos sobre el ciclo celular de los queratinocitos, lo cual podría promover alteraciones en la mucosa oral y participar como un factor etiológico agregado $^{62}$. Todos estos factores unidos a los efectos antiangiogénicos de los bifosfonatos ${ }^{14}$ favorecen la osteonecrosis.

Además de las infecciones de origen dentario y periodontal marginal, los factores traumáticos y la exposición a bifosfonatos, la adición de estos a quimioterapia y el uso de esteroides (dexametasona y prednisona) ${ }^{21,55}$ en estos pacientes pueden también afectar los procesos de reparación y regeneración ósea y actuar de manera sinérgica en la inducción de esta complicación terapéutica; por lo tanto, todos ellos deben ser considerados como posibles cofactores etiológicos de osteonecrosis.

Estudios histopatológicos $22,32,47$ han demostrado la presencia de Actinomyces en contacto con el tejido óseo vital remanente en las osteomielitis en pacientes tratados con bifosfonatos. Hansen et al. ${ }^{47}$, en 8 muestras, citan que todas presentaron al examen microscópico Actinomyces; asimismo observó la presencia de numerosos osteoclastos en contacto cercano al tejido óseo vital en el $62,5 \%$ de ellas. La presencia de osteoclastos en los sitios de reabsorción ósea sugiere que estas células están implicadas en los mecanismos de osteólisis. Es interesante observar que el aumento en el número de osteoclastos y la evidencia histológica de su actividad se encuentran en pacientes tratados con fármacos empleados para inhibir la función osteoclástica e inducen apoptosis en estos. En este contexto, modelos previos de interacción entre osteoblastos, citoquinas y bacterias en la inducción de los mecanismos osteolíticos podrían explicar estos sucesos ${ }^{63}$.

Exodoncias previas a la instauración de la osteonecrosis son el antecedente odontológico más importante (62,9\%); las series revisadas abarcan desde un $33 \%^{33}$ hasta un $86 \%^{21}$; este valor indica que un alto porcentaje de casos podría ser desencadenado por maniobras quirúrgicas odontológicas. Sin embargo, el 36,2\% de los casos aparecen de manera "espontánea". En esta revisión, de los 214 casos asociados a exodoncias previas, el 64 \% se localizó en la mandíbula.
No se han establecido protocolos de tratamiento estandarizados, que dependen, hasta el momento, de cada caso individual, de su evolución y de la decisión del equipo médico-odontológico responsable. La terapia farmacológica se basa en la antibioterapia (amoxicilina-clavulánico, claritromicina o eritromicina) y aplicación tópica de geles de clorhexidina al $0,12 \%$. Los tratamientos quirúrgicos abarcan desde: desbridamiento, secuestrectomía, maxilectomía y mandibulectomía parcial o total ${ }^{19,20}$.

En resumen y para concluir, la terapéutica actual en el tratamiento del mieloma múltiple y del cáncer de mama, que implique el uso de bifosfonatos intravenosos, pamidronato y ácido zoledrónico, conlleva el riesgo potencial de desarrollar osteonecrosis de los maxilares. También se han publicado casos asociados al uso de alendronato oral, especialmente en pacientes con osteoporosis. Los cofactores de riesgo asociados son variados: infecciones orales, traumas, quimioterapia, corticoides, etc. Es importante reconocer esta nueva complicación e informarla en la comunidad médica y odontológica para establecer pautas en relación al diagnóstico, prevención y manejo terapéutico. Asimismo, el avance y desarrollo de los conocimientos en la identificación de las múltiples interacciones existentes en la generación de esta complicación permitirán comprender los mecanismos etiopatogénicos exactos involucrados.

\section{BIBLIOGRAFÍA}

1. Sung EC, Chan SM, Sakurai K, Chung E. Osteonecrosis of the maxilla as a complication to chemotherapy: a case report. Spec Care Dentist 2002;22:142-6.

2. Hui L, Wiernik PH. Avascular Necrosis of bone after adult acute lymphocytic leukemia treatment with methotrexate, vincristine, L-asparaginase and dexamethasone (MOAD). Am J Hematol 1996;52:184-8.

3. Thorn JJ, Hansen HS, Specht L, Bastholt L. Osteoradionecrosis of the jaws: clinical characteristics and relation to the field of irradiation. $J$ Oral Maxillofac Surg 2000;58:1088-93. 
4. Reuther T, Schuster T, Mende U, Kübler A. Osteoradionecrosis of the jaws as a side effect of radiotherapy of head and neck tumour patientsa report of a thirty year retrospective review. Int $\mathrm{J}$ Oral Maxillofac Surg 2003;32:289-95.

5. Marx RE. Pamidronate (Aredia) and Zoledronate (Zometa) induced avascular necrosis of the jaws: a growing epidemic. J Oral Maxillofac Surg 2003;61:1115-8.

6. Wang J, Goodger NM, Pogrel MA. Osteonecrosis of the jaws associated with cancer chemotherapy. J Oral Maxillofac Surg 2003;61:1104-7.

7. Migliorati CA. Bisphosphonates and oral cavity avascular bone necrosis. J Clin Oncol 2003;21: 4253-4.

8. Tanvetyanon T, Stiff PJ. Management of the adverse effects associated with intravenous bisphosphonates. Ann Oncol 2006;17:897-907.

9. Licata AA. Discovery, Clinical Development, and Therapeutic Uses of Bisphosphonates. Ann Pharmacother 2005;39:668-77.

10. Rogers MJ, Gordon S, Benford HL, Coxon FP, Luckman SP, Monkkonen J, Frith JC. Cellular and molecular mechanisms of action of bisphosphonates. Cancer 2000;86:2961-78.

11. Vitté C, Fleisch H, Guenther HL. Bisphosphonates induce osteoblasts to secrete an inhibitor of osteoclast-mediated resorption. Endocrinology 1996;137:2324-33.

12. Hughes DE, Wright KR, Uy HL, Sasaki A, Yoneda T, Roodman GD, Mundy GR, Boyce BF. Bisphosphonates promote apoptosis in murine osteoclasts in vitro and in vivo. J Bone Miner Res 1995; 10:1478-87.

13. Plotkin LI, Agurre JI, Kousteni S, Manolagas SC, Bellido T. Bisphosphonates and Estrogens Inhibit Osteocyte Apoptosis via Distinct Molecular Mechanisms Downstream of Extracellular Signalregulated Kinase Activation. J Biol Chem 2005;280:7317-25.
14. Wood J, Bonjean K, Ruetz S, Bellahcéne A, Devy L, Foidart JM. Castronovo V, Green JR. Novel antiangiogenic effects of the bisphosphonate compound zoledronic acid. J Pharmacol Exp Ther 2002; 302:1055-61.

15. Lee MV, Fong EM, Singer FR, Guenette RS. Bisphosphonate treatment inhibits the growth of prostate cancer cells. Cancer 2001;61:2602-8.

16. Boissier S, Magnetto S, Frappart L, Cuzin B, Ebetino FH, Delmas PD, Clezardin P. Bisphosphonates inhibit prostate and breast carcinoma cell adhesion to unmineralized and mineralized bone extracellular matrices. Cancer 1997;57:3890-4.

17. Tassone P, Tagliaferri P, Viscomi C. Zoledronic acid induces antiproliferative and apoptotic effects in human pancreatic cancer cells in vitro. $\mathrm{Br} \mathrm{J}$ Cancer 2003;88:1971-8.

18. Boissier S, Ferreras M, Peyruchaud O, Magnetto S, Ebetino FH, Colombel M, Delmas P, Delaisse JM, Clezardin P. Bisphosphonates inhibit breast and prostate carcinoma cell invasion, an early event in the formation of bone metastases. Cancer Res 2000;60:2949-54.

19. Expert Panel Recommendations for the Prevention, Diagnosis, and Treatment of Osteonecrosis of the Jaws: June 2004. Professional Education Material. East Hanover, NJ: Novartis; 2004.

20. Migliorati Ca, Casiglia J, Epstein J, Jacobsen PL, Siegel MA, Woo SB. Managing the care of patients with bisphosphonate-associated osteonecrosis. Journal American Dental Association 2005;136: 1658-68.

21, Ruggiero SL, Mehrotra B, Rosenberg TJ, Engroff SL. Osteonecrosis of the jaws associated with the use of bisphosphonates: a review of 63 cases. J Oral Maxillofac Surg 2004;62:52734.

22. Lugassy G, Shaham R, Nemets A, Ben-Dor D, Nahlieli O. Severe osteomyelitis of the jaw in long- 
Escobar López EA, López López J, Marques Soares MS, Chimenos Küstner E. Osteonecrosis de los maxilares asociada a bifosfonatos: revisión sistemática

term survivors of multiple myeloma: a new clinical entity. Am J Med 2004;117:440-1.

23. Thakkar SG, Isada C, Englund K, Tomford W, Karam MA, Reed JL, Smith J, Hussein MA. Bisphosphonate therapy associated with an increased incidence of mandibular/maxillary osteomyelitis in multiple myeloma patients. Blood 2004; 104:4925.

24. Schuster MW, Dymek JM. Oral cavity avascular bone necrosis a newly recognized complication of intravenous (iv) bisphosphonate therapy in cancer patients. Blood 2004; 104:4905.

25. Pires FR, Miranda A, Cardoso ES, Cardoso AS, Fregnani ER, Pereira CM, Correa ME, Almeida JP, Alves Fde A, Lopes MA, de Almeida OP. Oral avascular bone necrosis associated with chemotherapy and bisphosphonate therapy. Oral Dis 2005;11:365-9.

26. Bagán JV, Jiménez Y, Murillo J, Hernández S, Poveda R, Sanchís JM, Díaz JM, Scully C. Jaw osteonecrosis associated with bisphosphonates: multiple exposed areas and its relationship to teeth extractions. Study of 20 cases. Oral Oncol 2006;42:327-9.

27. Gibbs SD, O'Grady J, Seymour JF, Prince HM. Bisphosphonate-induced osteonecrosis of the jaw requires early detection and intervention. Med $\mathrm{J}$ Aust 2005; 183:549-50.

28. Merigo E, Manfredi M, Meleti M, Corradi D, Vescovi P. Jaw bone necrosis without previous dental extractions associated with the use of bisphosphonates (pamidronate and zoledronate): a four-case report. J Oral Pathol Med 2005;34: 613-7.

29. Carter G, Goss AN, Doecke C. Bisphosphonates and avascular necrosis of the jaw: a possible association. Med J Aust 2005;182:413-5.

30. Lenz JH, Steiner-Krammer B, Schmidt W, Fietkau R, Mueller PC, Gundlach KK. Does avascular necrosis of the jaws in cancer patients only occur following treatment with bisphos- phonates? J Craniomaxillofac Surg 2005;33: 395-403.

31. Ficarra G, Beninati F, Rubino I, Vannucchi A, Longo G, Tonelli P, Pini Prato G. Osteonecrosis of the jaws in periodontal patients with a history of bisphosphonates treatment. J Clin Periodontol 2005;32:1123-8.

32. Melo MD, Obeid G. Osteonecrosis of the jaws in patients with a history of receiving bisphosphonate therapy: strategies for prevention and early recognition. J Am Dent Assoc 2005;136: 1675-81.

33. Migliorati CA, Schubert MM, Peterson DE, Seneda LM. Bisphosphonate-associated osteonecrosis of mandibular and maxillary bone: an emerging oral complication of supportive cancer therapy. Cancer 2005; 104:83-93.

34. Olson KB, Hellie CM, Pienta KJ. Osteonecrosis of jaw in patient with hormone refractory prostate cancer treated with zoledronic acid. Urology 2005; 66:658.e1-658.e3.

35. Purcell PM, Boyd IW. Bisphosphonates and osteonecrosis of the jaw. Med J Aust 2005; 182:417-8.

36. Vannucchi AM, Ficarra G, Antonioli E, Bosi A. Osteonecrosis of the jaw associated with zoledronate therapy in a patient with multiple myeloma. Br J Haematol 2005;128:738.

37. Katz H. Endodontic implications of bisphosphonate-associated osteonecrosis of the jaws: a report of three cases. J Endod 2005;31:831-4.

38. Sarathy AP, Bourgeois SL Jr, Goodell GG. Bisphosphonate-associated osteonecrosis of the jaws and endodontic treatment: two case reports. J Endod 2005;31:759-63.

39. Schirmer I, Peters H, Reichart PA, Durkop H. Bisphosphonates and osteonecrosis of the jaw. Mund Kiefer Gesichtschir 2005;9:239-45.

40. Guarneri V, Donati S, Nicolini M, Giovannelli S, D’Amico R, Conte PF. Renal Safety and Efficacy 
of i.v. Bisphosphonates in Patients with Skeletal Metastases Treated for up to 10 Years. Oncologist 2005; 10:842-8.

41. Markiewicz MR, Margarone JE, Campbell JH, Aguirre A. Bisphosphonate-associated osteonecrosis of the jaws: a review of current knowledge. J Am Dent Assoc 2005;136:1669-74.

42. Bamias A, Kastritis E, Bamia C, Moulopoulos LA, Melakopoulos I, Bozas G, Koutsoukou V, Gika D, Anagnostopoulos A, Papadimitriou C, Terpos E, Dimopoulos MA. Osteonecrosis of the jaw in cancer after treatment with bisphosphonates: incidence and risk factors. J Clin Oncol 2005; 23:8580-7.

43. Dimitrakopoulos I, Magopoulos C, Karakasis D. Bisphosphonate-induced avascular osteonecrosis of the jaws: a clinical report of 11 cases. Int $\mathrm{J}$ Oral Maxillofac Surg 2006;35:588-93.

44. Mignogna MD, Lo Russo L. Osteonecrosis of the Jaws Associated With Bisphosphonate Therapy. J Clin Oncol 2006;24:1475-7.

45. Badros A, Weikel D, Salama A, Goloubeva O, Schneider A, Rapoport A, Fenton R, Gahres N, Sausville E, Ord R, Meiller T. Osteonecrosis of the Jaw in Multiple Myeloma Patients: Clinical Features and Risk Factors. Journal of Clinical Oncology 2006;24:945-52.

46. Farrugia MC, Summerlin DJ, Krowiak E,Huntley T, Freeman S, Borrowdale R, Tomich C. Osteonecrosis of the Mandible or Maxilla Associated with the use of New Generation Bisphosphonates. Laryngoscope 2006;116:115-20.

47. Hansen T, Kunkel M, Weber A, Kirkpatrick CA. Osteonecrosis of the jaws in patients treated with bisphosphonates - histomorphologic analysis incomparison with infected osteoradionecrosis. J Oral Pathol Med 2006;35:155-60.

48. Pastor-Zuazaga D, Garatea-Crelgo J, MartinoGorbea R, Etayo-Pérez A, Sebastián-López C. Osteonecrosis of the jaws and bisphosphonates. Report of three cases. Med Oral Patol Oral Cir Bucal 2006; 11:E76-9.
49. Zarychanski R, Elphee E, Walton P, Johnston J. Osteonecrosis of the jaw associated with pamidronate therapy. Am J Hematol 2006;81: 73-5.

50. Carneiro E, Vibhute P, Montazem A, Som PM. Bisphosphonate-Associated Mandibular Osteonecrosis. Am J Neuroradiol 2006;27:1096-7.

51. Soileau KM. Oral post-surgical complications following the administration of bisphosphonates given for osteopenia related to malignancy. J Periodontol 2006; 77:738-43.

52. González-Moles MA, Bagán-Sebastián JV. Alendronate-related oral mucosa ulcerations. J Oral Pathol Med 2000;29:514-8.

53. Demerjian N, Bolla G, Spreux A. Severe oral ulcerations induced by alendronate. Clin Rheumatol. 1999; 18:349-50.

54. Krasagakis K, Krüger-Krasagakis S, Ioannidou D, Tosca A. J Am Acad Dermatology 2004;50: 671-2.

55. Durie BG, Katz M, Crowley J. Osteonecrosis of the jaw and bisphosphonates. N Engl J Med 2005; 353:99-102.

56. Estilo CS, Van Poznak CH, Williams T, Evtimovska E, Tkach L, Halpern JL, et al. Osteonecrosis of the maxilla and mandible in patients treated with bisphosphonates: a retrospective study. Proc Am Soc Clin Oncol 2004;22:750.

57. Tarassoff P, Csermak K. Avascular necrosis of the jaws: risk factors in metastatic cancer patients. J Oral Maxillofac Surg 2003;61:1238-9.

58. Reid IR, Brown JP, Burckhardt P, Horowitz Z, Richardson P, Trechsel $U$ et al. Intravenous zoledronic acid in postmenopausal women with low bone mineral density. N Engl J Med 2002;346:653-61.

59. Mashiba T, Mori S, Burr DB, Komatsubara S, Cao Y, Manabe T, et al. The effects of suppressed bone remodeling by bisphosphonates on micro- 
damage accumulation and degree of mineralization in the cortical bone of dog rib. J Bone Miner Metab 2005;23 Suppl:36-42.

60. Polizzotto MN, Cousins V, Schwarer AP. Bisphosphonate-associates of the auditory canal $\mathrm{Br} \mathrm{J}$ Haematol 2005;132:114-7.

61. Parfett AM: Osteonal and hemiosteonal remodeling, the spatial and temporal framework for signal traffic in adult human bone. J Cellular Biochem 1994; 55:273.

62. Reszka AA, Halasy-Nagy J, Rodan GA. Nitrogenbisphosphonates block retinoblastoma phosphorylation and cell growth by inhibiting the cholesterol biosynthesis pathway in a keratinocyte model for esophageal irritation. Mol Pharmacol 2001;59:193-202.

63. Nair SP, Meghij S, Wilson M, Reddi K, White P, Henderson B. Bacterially induced bone destruction: mechanisms and misconceptions. Infect Immun 1996;64:2371- 80.

\section{CORRESPONDENCIA}

Eduardo Chimenos Küstner

Vía Augusta $124,1^{\circ} 3^{a}$

08006 Barcelona

C.E.:13598eck@comb.es 